\title{
COVID-19 in Children: Do They Have a Lower Risk of Severe Infection
} Than Adults?

\author{
Mohammad Reza Amiri (i) 1, * \\ ${ }^{1}$ Dr. Masih Daneshvari Hospital, Shahid Beheshti University of Medical Sciences, Tehran, Iran \\ "Corresponding author: Dr. Masih Daneshvari Hospital, Shahid Beheshti University of Medical Sciences, Tehran, Iran. Email: amiri.mza@gmail.com
}

Received 2020 October 13; Revised 2021 February 09; Accepted 2021 August 28.

\begin{abstract}
The novel coronavirus disease 2019 (COVID-19), which was first identified in Wuhan, China, in late 2019, has now spread around the world. It significantly affects the lower respiratory tract, and pneumonia is always present in patients with the severe form of the disease. Many studies have shown that the severity of COVID-19 is lower in pediatric populations. It is important to determine why infants and young children are not severely affected by COVID-19. By increasing our awareness of this disease, we can prioritize our limited health resources. Several theories have been proposed to explain such significant differences between the pediatric and adult populations with COVID-19. Some of them are: (1) the role of angiotensin-converting enzyme 2 (ACE2); (2) cross-immunity with other severe acute respiratory syndrome (SARS)-like viruses; (3) obtunded systemic inflammatory response in children; (4) more efficient T cells in them; (5) interactions of SARS-Coronavirus-2 (SARS-CoV-2) with other viruses in the mucosa of the lungs and airways; and (6) a hypothesis that COVID-19 proteins onslaught the heme on the $\beta-1$ chain of hemoglobin. The important thing to keep in mind is that the asymptomatic and mildly symptomatic pediatrics are crucial in the spread of COVID-19.
\end{abstract}

Keywords: COVID -19, SARS-CoV-2 in Children, ACE2 in COVID 19 Pneumonia

\section{Context}

The novel Coronavirus disease 2019 (COVID-19), which was first identified in Wuhan, China, in late 2019, has spread around the world. The World Health Organization (WHO) declared this disease as pandemic on March 11, 2020 (1). COVID-19 significantly affects the lower respiratory tract, and pneumonia is always present in patients with the severe form of the disease (2). Human-to-human transmission is the main route of transmission, which occurs through aerosols, respiratory droplets, direct contact, and fecal-oral transmission. So far, vertical transmission of COVID-19 has not been confirmed; however, there are reports of affected neonates born to COVID-19 mothers (3).

It is not clear whether infants and young children are at a lower risk of severe COVID-19. To answer this question, a literature search was carried out in Medline, PubMed, EMBASE, Scopus, and Web of Science databases. The following terms and keywords were used in our searches: "COVID19 in children"; "comparison of the severity of COVID-19 in children and adults"; "clinical characteristics of COVID19"; "SARS-CoV infection"; and "immunopathogenesis of COVID-19". In this study, no ethical approval or patient consent was required. The relevant papers with confirmed cases of SARS-CoV-2 between January1 and July1,2020, were included in this study. Most of the studies had been conducted in China.

Generally, the lifestyle of children is different from adults. They do not have outdoor activities and do not travel as much as adults; therefore, they are not at a high risk of infection. These observations can explain the lower frequency of COVID-19 in pediatric populations (4). Many studies have shown that the severity of COVID-19 is lower in newborns and pediatric populations, and the majority of these cases are detected via screening tests of infected families (3). In March 2020, Jonas at the Karolinska Institutet in Sweden reviewed 45 studies on COVID-19 among children (mostly in China) and reported that children developed a milder disease with a better prognosis and extremely low mortality (1). Moreover, Rahimzadeh et al. (2019) reported similar results in Iran (5).

In another study by Du et al. (2020) in China, a total of 67 patients with COVID-19 (53 adults and 14 children) were examined, and a lower rate of clinical disease was found among children (6). However, a few studies, including the study by DeBiasi et al. (2020), yielded inconsistent results (7). It is important to determine why infants and young children are not severely affected by COVID-19. On the other 
hand, by increasing our awareness of this disease, we can prioritize our limited health resources.

According to studies on pediatrics, some infectious diseases, such as paralytic polio and rubella, are less severe in children. Also, the mortality of severe respiratory distress syndrome (SARS) is lower in children and adults younger than 24 years (8). Generally, the immune system of children and adults differs in terms of composition and functional responsiveness (9). Overall, children have a healthier respiratory system and a more active innate immune system than adults. They are less exposed to air pollutants, and cigarette smoke compared to adults and have fewer underlying diseases (8). Several theories have been proposed to explain such significant differences between the pediatric and adult populations with COVID-19, which are discussed below.

\section{Discussion}

\subsection{Role of Angiotensin-Converting Enzyme 2 (ACE2)}

Differences in the distribution, maturation and functioning of virus receptors can be a possible cause of the difference in the incidence of COVID-19 associated with age. In humans, ACE2 is considered a receptor for SARS-CoV-2 and human coronavirus NL63 $(4,10)$. The down-regulation of ACE2 due to aging and ethnicity has been reported in experiments on animal models and humans (3). In this regard, Rawat et al. (2020) stated that ACE2 was less mature in children; therefore, it could not act effectively as a receptor for COVID-19. Also, the intracellular reaction induced by ACE2 in alveolar epithelial cells has been shown to be lower in children compared to adults. Overall, the lower respiratory tract cells, which are commonly affected by COVID-19, are richer in ACE2. In children, COVID-19 more affects the upper respiratory tract than the lower respiratory tract (9). Nevertheless, some researchers believe that ACE2 is associated with the protective mechanisms of the lungs and protects them against severe damage due to sepsis, SARS, acid aspiration, and lethal influenza (4).

\subsection{Cross-Immunity with Other SARS-Like Viruses}

The immunity caused by viruses (e.g., coronaviruses) can be protective for the pediatric population (9). There may be a high level of cross-reactivity between antibodies generated against the relevant structural viral proteins. The absence of severe COVID-19 in areas with a high prevalence of SARS-like infections may be attributed to partial immunity due to the cross-reactivity of T-cell and B-cell epitopes, as well as antibodies (11). Anderson et al. (2020) used SARS convalescent sera to neutralize SARS-CoV-2. They found that antibodies generated against SARS-CoV exhibited SARS-CoV-2 cross-neutralization properties; however, in this study, the cross-neutralization level of SARS survivors' sera against SARS-CoV-2 was not sufficient for passive immunotherapy of COVID-19 patients (12).

On average, children up to six years of age acquire upper respiratory tract infections (URIs) eight to 12 times a year, while adolescents and adults acquire URIs two to four times a year $(13,14)$. Our knowledge of other coronavirusassociated diseases (SARS and MERS) suggests that previous exposure to other respiratory viruses may make children's immune systems more resilient $(9,15)$.

\subsection{The Obtunded Systemic Inflammatory Response}

Several studies have shown that patients with serious SARS-COV-2 suffer from a "cytokine storm" (CS). This is a critical and serious condition defined by hyperferritinemia, severe systemic inflammation, hemodynamic instability, acute respiratory distress syndrome (ARDS), and multiorgan failure. CS needs intensive care admission, and treatment should begin promptly; otherwise, it may lead to death. The trigger for CS is an uncontrolled immune response leading to the continuous activation of immune cells and macrophages. These cells generate excessive amounts of cytokines, resulting in a CS. Clinical findings of CS are attributed to the activity of pro-inflammatory cytokines, such as interleukin-1 (IL-1), IL-6, IL-18, interferongamma (IFN- $\gamma$ ), and tumor necrosis factor-alpha (TNF- $\alpha$ ) (16).

Inflammatory reactions vary in adult and pediatric patients during their lifetime (17). Wynn et al. (2011) showed a marked difference in the transcriptomic response associated with age in children with septic shock (18). According to a study by Schouten et al. (2019), an age-related increase in pro-inflammatory cytokines, linked to neutrophil function, has a relationship with the severity of ARDS and can partly explain the age-related difference (19). Some researchers suggest that CS is underdeveloped in children (9).

These different responses could explain the Centers for Disease Control and Prevention (CDC) data, which revealed that only $54 \%$ of pediatric patients coughed compared to adults (80\%). Also, much lower rates of shortness of breath were found in children compared to adults (13 vs. 43\%) (9). According to the clinical data from Wuhan, China, the total count of peripheral blood lymphocytes gradually reduces in adults through an inflammatory reaction to SARSCoV-2, which results in the proliferation and spread of this virus. Generally, the white blood cell count and the absolute number of lymphocytes in the pediatric population are normal because of the incomplete development of natural immunity (20). It is critical to gather more informa- 
tion about differences in immune reactions in different age groups for targeted immunotherapies.

\subsection{More Efficient T-Cells}

$\mathrm{T}$ cells are crucial to clear viruses in mice affected by SARS-CoV (9). Le Bert et al. (2020) examined 36 individuals convalescing from SARS-CoV-2 and found that in all of them, CD4 and CD8 T cells identified several regions of the $\mathrm{N}$ protein of the virus. The researchers realized that 23 patients developing and recovering from SARS infection in 2003 had long-lasting memory T cells able to react strongly with the $\mathrm{N}$ protein of SARS-CoV after 17 years. Also, they found that in people who had no previous or current history of SARS-CoV-2/COVID-19 infection or had no contact with other people with these viruses, SARS-CoV-2 specific $\mathrm{T}$ cells frequently targeted the NSP7, NSP13, and N protein of SARS-CoV-2 $(9,21)$.

Certainly, more studies in this area are required for the SARS-CoV-2 pandemic management.

\subsection{Virus-to-Virus Interactions}

Some researchers suggest that the simultaneous presence of other viruses in the mucosa of the lungs and airways, which is common in young children, can limit the growth of SARS-CoV-2 by direct virus-to-virus interactions and competition (22-26). Nickbakhsh et al. (2019) statistically analyzed a collection of diagnostic reports collected over several years from 11 types of respiratory viruses, including the human coronavirus. The analysis showed that there was an interaction between respiratory viruses, which was statistically provable. Using computer simulations, they understood that very short-lived interferences could explain why cold infections were less likely to occur during flu outbreaks (22).

\subsection{Fetal Hemoglobin}

Newborns have $80 \%$ fetal hemoglobin content, composed of alpha and gamma chains (27). Some researchers hypothesize that SARS-CoV-2 proteins (orfiab, ORF10, and ORF3a) attack the heme of the hemoglobin $1-\beta$ chain, dissociate iron, and form porphyrin. Such attacks can reduce hemoglobin, resulting in hypoxia, and also inhibit the normal heme metabolic pathway (9). However, this hypothesis has not been approved, and further research is needed in the future.

\section{Conclusions}

COVID-19 can develop in children with mild or no symptoms. Discovering the mysterious aspects of this disease in children, we can provide critical information about both optimal immune system targeting and viral function. The asymptomatic and mildly symptomatic pediatric populations contribute to the spread of SARS-CoV-2. Therefore, future studies in this area can help researchers develop prophylactic and therapeutic strategies to combat this lethal disease.

\section{Footnotes}

Authors' Contribution: Study concept and design, M.A; Acquisition of data, M.A; Analysis and interpretation of data, M.A; Drafting of the manuscript, M.A; Critical revision of the manuscript for important intellectual content, M.A; Statistical analysis, M.A; Administrative, technical, and material support, M.A; Study supervision, M.A.

Conflict of Interests: The author does not have any potential conflict of interest for this manuscript.

Funding/Support: I did not have any funding/support from an institution or organization to conduct this study.

\section{References}

1. Ludvigsson JF. Systematic review of COVID-19 in children shows milder cases and a better prognosis than adults. Acta Paediatr. 2020;109(6):1088-95. doi: 10.1111/apa.15270. [PubMed: 32202343]. [PubMed Central: PMC7228328].

2. Lai CC, Shih TP, Ko WC, Tang HJ, Hsueh PR. Severe acute respiratory syndrome Coronavirus 2 (SARS-CoV-2) and Coronavirus disease-2019 (COVID-19): The epidemic and the challenges. Int J Antimicrob Agents. 2020;55(3):105924. doi: 10.1016/j.ijantimicag.2020.105924. [PubMed: 32081636]. [PubMed Central: PMC7127800].

3. Seyedi SJ, Shojaeian R, Hiradfar M, Mohammadipour A, Alamdaran SA. Coronavirus disease 2019 (COVID-19) outbreak in pediatrics and the role of pediatricians: A systematic review. Iran J Pediatr. 2020;30(2). e102784. doi: 10.5812/ijp.102784.

4. Lee PI, Hu YL, Chen PY, Huang YC, Hsueh PR. Are children less susceptible to COVID-19? J Microbiol Immunol Infect. 2020;53(3):371-2. doi: 10.1016/j.jmii.2020.02.011. [PubMed: 32147409]. [PubMed Central: PMC7102573]

5. Rahimzadeh G, Ekrami Noghabi M, Kadkhodaei Elyaderani F, Navaeifar MR, Enayati AA, Manafi Anari A, et al. COVID-19 infection in Iranian children: A case series of 9 patients.JPediatr Rev. 2020;8(2):139-44. doi: 10.32598/jpr.8.2.139.

6. Du W, Yu J, Wang H, Zhang X, Zhang S, Li Q, et al. Clinical characteristics of COVID-19 in children compared with adults in Shandong Province, China. Infection. 2020;48(3):445-52. doi: 10.1007/s15010-02001427-2. [PubMed: 32301099]. [PubMed Central: PMC7161094].

7. DeBiasi RL, Song X, Delaney M, Bell M, Smith K, Pershad J, et al. Severe Coronavirus disease-2019 in children and young adults in the Washington, DC, Metropolitan region. J Pediatr. 2020;223:199-203 e1. doi: 10.1016/j.jpeds.2020.05.007. [PubMed: 32405091]. [PubMed Central: PMC7217783].

8. Kliegman RM, St Geme JW, Blum NJ, Shah SS, Takser RC, Wilson KM. Nelson textbook of pediatrics. Philadelphia, USA: Elsevier; 2020.

9. Rawat M, Chandrasekharan P, Hicar MD, Lakshminrusimha S. COVID19 in newborns and infants-low risk of severe disease: Silver lining or dark cloud? Am J Perinatol. 2020;37(8):845-9. doi: 10.1055/s-00401710512. [PubMed: 32380565]. [PubMed Central: PMC7356082]. 
10. Hofmann H, Pyrc K, van der Hoek L, Geier M, Berkhout B, Pohlmann S. Human coronavirus NL63 employs the severe acute respiratory syndrome coronavirus receptor for cellular entry. Proc Natl Acad Sci U S A. 2005;102(22):7988-93. doi: 10.1073/pnas.0409465102. [PubMed: 15897467]. [PubMed Central: PMC1142358].

11. Yaqinuddin A. Cross-immunity between respiratory coronaviruses may limit COVID-19 fatalities. Med Hypotheses. 2020;144:110049. doi: 10.1016/j.mehy.2020.110049. [PubMed: 32758887]. [PubMed Central: PMC7326438].

12. Anderson DE, Tan CW, Chia WN, Young BE, Linster M, Low JH, et al. Lack of cross-neutralization by SARS patient sera towards SARS-CoV-2. Emerg Microbes Infect. 2020;9(1):900-2. doi: 10.1080/22221751.2020.1761267. [PubMed: 32380903]. [PubMed Central: PMC7241448].

13. Chen IM, Loh JP, Chuah CXP, Gao QHC, Sun Y, Ng SH, et al. Evidence for cross-protection against subsequent febrile respiratory illness episodes from prior infections by different viruses among Singapore military recruits 2009-2014. J Infect Dis. 2019;219(12):1913-23. doi: 10.1093/infdis/jiz046. [PubMed: 30722024]. [PubMed Central: PMC6534195].

14. Weintraub B. Upper respiratory tract infections. Pediatr Rev. 2015;36(12):554-6. doi: 10.1542/pir.36-12-554. [PubMed: 26628739].

15. Li AM, Ng PC. Severe acute respiratory syndrome (SARS) in neonates and children. Arch Dis Child Fetal Neonatal Ed. 2005;90(6):F461-5. doi: 10.1136/adc.2005.075309. [PubMed: 16244207]. [PubMed Central: PMC1721969].

16. Ragab D, Salah Eldin H, Taeimah M, Khattab R, Salem R. The COVID19 cytokine storm; what we know so far. Front Immunol. 2020;11:1446. doi: 10.3389/fimmu.2020.01446. [PubMed: 32612617]. [PubMed Central: PMC7308649].

17. Molloy EJ, Bearer CF. COVID-19 in children and altered inflammatory responses. Pediatr Res. 2020;88(3):340-1. doi:10.1038/s41390-020-0881y. [PubMed: 32244248].

18. Wynn JL, Cvijanovich NZ, Allen GL, Thomas NJ, Freishtat RJ, Anas N, et al. The influence of developmental age on the early transcriptomic response of children with septic shock. Mol Med.2011;17(11-12):1146-56. doi: 10.2119/molmed.2011.00169. [PubMed: 21738952]. [PubMed Cen- tral: PMC3321808].

19. Schouten LR, van Kaam AH, Kohse F, Veltkamp F, Bos LD, de Beer FM, et al. Age-dependent differences in pulmonary host responses in ARDS: A prospective observational cohort study. Ann Intensive Care. 2019;9(1):55. doi: 10.1186/s13613-019-0529-4. [PubMed: 31089908]. [PubMed Central: PMC6517452].

20. Dong Y, Mo X, Hu Y, Qi X, Jiang F, Jiang Z, et al. Epidemiology of COVID-19 among children in China. Pediatrics. 2020;145(6). doi: 10.1542/peds.2020-0702. [PubMed: 32179660].

21. Le Bert N, Tan AT, Kunasegaran K, Tham CYL, Hafezi M, Chia A, et al. SARS-CoV-2-specific $\mathrm{T}$ cell immunity in cases of COVID-19 and SARS, and uninfected controls. Nature. 2020;584(7821):457-62. doi: 10.1038/s41586-020-2550-z. [PubMed: 32668444].

22. Nickbakhsh S, Mair C, Matthews L, Reeve R, Johnson PCD, Thorburn $\mathrm{F}$, et al. Virus-virus interactions impact the population dynamics of influenza and the common cold. Proc Natl Acad Sci U S A. 2019. doi: 10.1073/pnas.1911083116. [PubMed: 31843887]. [PubMed Central: PMC6936719].

23. Brodin P. Why is COVID-19 so mild in children? Acta Paediatr 2020;109(6):1082-3. doi: 10.1111/apa.15271. [PubMed: 32212348].

24. Linde A, Rotzen-Ostlund M, Zweygberg-Wirgart B, Rubinova S, Brytting M. Does viral interference affect spread of influenza? Euro Surveill. 2009;14(40). [PubMed: 19822124].

25. Casalegno JS, Ottmann M, Duchamp MB, Escuret V, Billaud G, Frobert E, et al. Rhinoviruses delayed the circulation of the pandemic influenza A (H1N1) 2009 virus in France. Clin Microbiol Infect. 2010;16(4):326-9. doi: 10.1111/j.1469-0691.2010.03167.x. [PubMed: 20121829].

26. Opatowski L, Baguelin M, Eggo RM. Influenza interaction with cocirculating pathogens and its impact on surveillance, pathogenesis, and epidemic profile: A key role for mathematical modelling. PLoS Pathog. 2018;14(2). e1006770. doi: 10.1371/journal.ppat.1006770. [PubMed: 29447284]. [PubMed Central: PMC5814058].

27. Wong P, Weerakul J, Sritippayawan S. Hemoglobin analysis in the first year of life. Mediterr J Hematol Infect Dis. 2016;8(1). e2016012. doi: 10.4084/MJHID.2016.012. [PubMed: 26977271]. [PubMed Central: PMC4771140]. 